\title{
Bioanalysis
}

\section{Demonstrating biosimilar and originator antidrug antibody binding comparability in antidrug antibody assays: a practical approach}

\begin{abstract}
Biosimilar drug development has brought new challenges to bioanalytical ligandbinding assays used to determine drug concentration, antidrug antibodies and neutralizing antibodies. One particular challenge is how to demonstrate that the antidrug antibody assay can adequately detect antibodies against both biosimilar and originator. In this paper, we review the current guidelines and literature for practical recommendations and present a gap analysis. Case examples of antibody binding comparability testing are presented, and the challenges and implications are discussed. Based on the lessons learned from our biosimilar assay applications, we recommend a bioanalytical comparability testing approach that is outlined and discussed.
\end{abstract}

First draft submitted: 15 May 2017; Accepted for publication: 12 July 2017;

Published online: 18 September 2017

Keywords: ADA • antigenicity • biosimilar $\bullet$ comparability $\bullet$ immunogenicity $\bullet$ one-assay

- practical • validation

\section{Background \& scope}

Biosimilars are often called follow-on biologics and are intended to be a similar version of the originator drug that is already marketed. Biosimilars are complex proteins and distinct from the small-molecule generics [1]. With the ongoing patent expiry of a number of biologics, biosimilars offer an interesting new commercial opportunity to pharma companies. There are also key benefits where the main is that biosimilars offer access to alternative treatment modalities for patient groups which in the past have not been offered a treatment opportunity. Regulators are positive about the concept and have developed legislation and guidance's for the development of biosimilars [2-5]. A critical aspect of development is to demonstrate similarity of physiochemical properties [6]. However, similarity must also be demonstrated through nonclinical and clinical studies; hence, the bioanalytical assays (pharmacokinetic [PK] assay, antidrug antibody [ADA] assay and neu- tralizing antibody assays) must be able to detect both the biosimilar and originator drug molecules in a comparable manner in order to be able to accurately assist in demonstrating that there are no meaningful differences in PK and immunogenicity. Bioanalytical biosimilar testing recommendations for the PK assay are extensively discussed in literature, and most of the industry take inspiration from the practical recommendations of Marini et al. [7]. For the immunogenicity assays, the practical approach is neither yet well defined nor is there a full consensus in the industry. The testing terminology related to one-assay justification or bioanalytical assay comparability is also quite varied in literature. Various terms have been used (e.g., antigenicity, drug comparability, antigenic equivalence and titration testing), yet we suggest the term 'ADA binding comparability' testing. We believe that this term describes best the characteristics of the experiment and observations obtained. The scope of this paper is
Janka Ryding*,1, Michael Stahl ${ }^{1}$ \& Martin Ullmann ${ }^{1}$ 'Fresenius-Kabi (SwissBiosim), Z.I. de l'Ouriettaz, 1170 Aubonne, Switzerland

*Author for correspondence: janka.ryding@fresenius-kabi.com newlands press part of 
to review current guidelines and literature for practical recommendations and to present a gap analysis. In addition, case examples of ADA binding comparability testing are presented, and the challenges and implications are discussed. Finally, we propose a practical and pragmatic approach to ADA assay comparability testing using the one-assay approach.

\section{Literature review \& gap analysis}

A review of the current recommendations and definitions is presented below and constitutes the base of the gap analysis performed in this publication.

There are several regulatory guidance documents from the WHO, the EMA and the US FDA on biosimilar drug development [2-5], as well as regulatory guidance and extensive publications on PK and immunogenicity immunoassay validation for biologics in general [8-13]. But when it comes to the practical conduct of immunogenicity studies in the bioanalytical biosimilar context, the literature is sparse (Table 1) [14-20].

Probably the most debated aspect in the field and in the literature is the choice between a one-assay and a two-assay approach. Each approach has its own promoters (Table 1), advantages and disadvantages [15]. The concept of a two-assay approach means using two assays, one with biosimilar-labeled drug and one with originator-labeled drug to detect ADAs against biosimilar and originator $[15,19]$, whereas in the one-assay approach one set of labeled reagents (usually biosimilar drug) is used. The two-assay approach could be considered to potentially offer a more representative picture of the immunogenicity of the biosimilar and originator, but the approach has significant challenges both operationally (e.g., how to manage unblinding) and from a data evaluation perspective. In other words, how can two datasets coming from two distinct assays support that the immunogenicity profiles of the biosimilar and the originator are similar. In contrast, the one-assay approach logically minimizes this comparative assay bias and simplifies data evaluation. Samples will not need to be analyzed twice (once with each assay) or requiring unblinding. On the other hand, the higher risk of false-negative results (i.e., potentially risk missing epitopes that are unique for the originator) is frequently being raised as a concern. This could be considered as an acceptable risk when using the biosimilar as a capture agent only the immunogenicity of the originator might be affected by false-negative results and hence underestimated [18]. Chamberlain suggest a middle ground where the oneassay approach is applied, but study samples are then inhibited with both biosimilar and originator [17]. The variability of a two-assay setup is circumvented, and biosimilar potential novel epitopes can, in principle, be detected, but challenges remain on cut-point applications and interpretation of data.

Although the debate is not yet closed, recently the preference of a one-assay approach has been reported as a consensus [14,21]. In the remaining part of this section, we will focus on the recommendations specific to the one-assay approach.

A central question to immunogenicity study design is the nature of the PC. The opinions on this topic in the literature are divergent (Table 1). The most conservative approach described is the usage of antibiosimilar and antioriginator positive controls (PCs) throughout the study from assay development until sample analysis [15,19]. Regrettably, it is not described how to integrate those PCs as criteria for the individual runs. Another described approach is to evaluate antibiosimilar and antioriginator PCs during development and, if comparable, to include only antibiosimilar PC during validation and analysis [20]. Finally, one author considers that the inclusion or evaluation of antibiosimilar and antioriginator PCs is not necessary and does not provide any added value due to the surrogate nature of polyclonal antibodies [17,18]. It is noteworthy that the usage of commercially available antibodies is mentioned and might constitute a viable option as system sustainability control during sample analysis [17].

There is a broad consensus that if the one-assay approach is chosen to compare the immunogenicity profiles of the biosimilar and the originator; additional experiments are required to confirm the suitability of the assay to detect both biosimilar and originator ADAs (Table 1). Unfortunately, there is currently no unanimity on the precise type of experiment that would constitute a sufficient proof. Cai et al. suggested dose-response experiments using antibiosimilar and antioriginator PCs $[19,20]$. In order to claim comparability, two requirements were to be fulfilled: the resulting curves should overlay with each other or at least be parallel, and the detection sensitivity for both PCs should be within twofold or threefold of each other $[19,20]$. Additionally, drug tolerance using antibiosimilar and antioriginator PCs and increasing concentrations of the respective drug should be assessed and should also be within a twofold or threefold range of each other $[19,20]$. Similarly Liu et al. highlights sensitivity and drug tolerance as being key to justify the one-assay approach [15]. As mentioned earlier, Chamberlain does not consider both antibiosimilar and antioriginator PCs as required; hence, the experiment that he proposes is designed with a single PC. In the described experiment, the PC should be titrated with increasing amount of biosimilar and originator drugs. 


\begin{tabular}{|c|c|c|c|c|c|}
\hline \multirow[t]{2}{*}{ Publication (year) } & \multirow{2}{*}{$\begin{array}{l}\text { Recommendation: one- } \\
\text { assay or two-assays }\end{array}$} & \multicolumn{4}{|c|}{ One-assay-specific recommendations } \\
\hline & & $\begin{array}{l}\text { Detection } \\
\text { reagent }\end{array}$ & PC & $\begin{array}{l}\text { Comparability } \\
\text { experiments }\end{array}$ & $\begin{array}{l}\text { When to assess } \\
\text { comparability }\end{array}$ \\
\hline Cai et al. (2011) & One assay & $\begin{array}{l}\text { Labeled } \\
\text { biosimilar }\end{array}$ & $\begin{array}{l}\text { Antibiosimilar and } \\
\text { antioriginator PCs }\end{array}$ & $\begin{array}{l}\text { Dose response } \\
\text { Sensitivity } \\
\text { Drug tolerance }\end{array}$ & - \\
\hline Cai et al. (2012) & Indeterminate & $\begin{array}{l}\text { Labeled } \\
\text { biosimilar }\end{array}$ & $\begin{array}{l}\text { Antibiosimilar and } \\
\text { antioriginator PCs }\end{array}$ & $\begin{array}{l}\text { Dose response } \\
\text { Sensitivity } \\
\text { Drug tolerance }\end{array}$ & Development \\
\hline $\begin{array}{l}\text { Chamberlain } \\
(2013)\end{array}$ & One assay & $\begin{array}{l}\text { Labeled } \\
\text { biosimilar }\end{array}$ & Biosimilar PC & $\begin{array}{l}\text { Signal inhibition } \\
\text { curves: using biosimilar } \\
\text { and originator }\end{array}$ & Validation \\
\hline $\begin{array}{l}\text { Chamberlain } \\
(2014)\end{array}$ & One assay & $\begin{array}{l}\text { Labeled } \\
\text { biosimilar }\end{array}$ & $\begin{array}{l}\text { Biosimilar or } \\
\text { originator PC } \\
\text { Commercially } \\
\text { sourced Ab } \\
\text { acceptable }\end{array}$ & $\begin{array}{l}\text { Signal inhibition } \\
\text { curves: using biosimilar } \\
\text { and originator }\end{array}$ & - \\
\hline Islam (2014) & Two assays & N/A & N/A & N/A & N/A \\
\hline Liu et al. (2015) & Indeterminate & - & $\begin{array}{l}\text { Biosimilar and } \\
\text { originator PCs }\end{array}$ & $\begin{array}{l}\text { Sensitivity } \\
\text { Drug tolerance }\end{array}$ & Development \\
\hline $\begin{array}{l}\text { Richards et al. } \\
\text { (2016) }\end{array}$ & One assay & $\begin{array}{l}\text { Labeled } \\
\text { biosimilar }\end{array}$ & - & $\begin{array}{l}\text { Signal inhibition } \\
\text { curves: using biosimilar } \\
\text { and originator }\end{array}$ & Development \\
\hline
\end{tabular}

The resulting inhibition curves should be overlapping $[17,18]$. A similar experiment is described in the tenth WRIB report in 2016 [21].

Although it seems that the one-assay approach using labeled biosimilar is emerging as a consensus, there is less convergence on resulting questions and how they are to be addressed: what are the minimal requirements in terms of experiments to claim comparable biosimilar and originator ADA binding and detection; which target criteria are to be met; when to perform these experiments during assay development or validation; and how to best document those experiments.

\section{Case studies: demonstrating the one-assay approach}

How can one demonstrate that the one-assay approach is suitable, in other words, that the assay in a comparable manner can detect antibodies against biosimilar and originator? Below is an outline of two biosimilar case studies and our lessons learned.

\section{Case study 1: monoclonal antibody}

The pharmaceutical drug type is a monoclonal antibody for a chronic disease. The bioanalytical strategy consisted of developing a tiered one-assay approach (labeled biosimilar was used for capture and detection) for ADA testing. Due to the recurring dosing, drug tolerance was a concern; hence, the assay utilized acid dissociation and a homogeneous bead approach, where the samples were preincubated with the biosimilar. The platform was based on electrochemiluminescence. The PC used was generated by hyperimmunization of goats with the biosimilar. Routinely, each assay included four negative control samples, two low concentration PCs and two high concentration PCs. All controls and samples were analyzed in duplicate. All comparisons were performed using biosimilar and originator US and EU lots.

During development, the following basic one-assay feasibility was investigated:

Relative antibody binding curves with titrated PC versus spiked confirmatory concentration of biosimilar/originator were compared. Experiments were performed at various minimum required dilutions, once per test scenario. Figure 1 illustrates one such example. The experiments demonstrated that at the confirmatory quench concentration, which, in principle, best mimics the actual in-study method application, ADAs across the titration range are equally detected in the 
confirmatory assay. A few different spiked confirmatory concentrations were assessed. Preferably, this should be performed with what is believed to be the final drug confirmatory concentration, in other words, late in development, to as much as possible mimic the final confirmatory assay. Performing the comparability testing at various minimum required dilutions could be considered excessive, but could also add interference information (which technically should already be available from other experiments). In this particular case, spiked response values were used in the evaluation as they are more consistent than inhibition percentages. One could also argue that response value evaluation is more sensitive to observing differences than any normalized value. The rationale was to provide a clear dataset for curve comparison. In all experiments, curve similarity was visually assessed.

Relative binding curves in an inhibition (drug-tolerance-like) experiment with biosimilar/originator were compared; that means, comparison with no PC and low $/ \mathrm{mid} /$ high PCs versus spiked/unspiked biosimilar/originator. The used PC concentrations were base estimates of suitable low $/ \mathrm{mid} / \mathrm{high}$ concentration levels. Experiments were performed at various minimum required dilutions, once per test scenario. Figure 2 illustrates one such example. The experiment demonstrated that regardless of drug concentration, low and high concentration ADAs exhibit a similar binding profile. Potentially, one could further claim that the binding profile is expected to be similar in the ADA concentrations between low and high PCs. Similarly, as for first development experiments, the drug-tolerance-like experiments were performed on a variety of PC concentrations and minimum required dilutions. As in the first development experiment, spiked response values were used in the evaluation. The experiments were performed with comparison data derived from multiple plates. In retrospect, ideally, the experiments should have been performed during the later development phase where better estimates of preliminary low $/ \mathrm{mid} /$ high PC concentrations were available. The comparison data should also be derived from the same plate; in other words, plate consolidation should be favored over the number of replicates. Overall during validation approximately ten experiments were performed related to this making the case for the one-assay approach. There were no particular indication of nonsimilarity that sparked the extensive testing but were more related to the normal quite conservative bioanalytical approaches that are often applied when no clear guidances are available.

During validation, the one assay-approach was verified with the following approach:

Drug tolerance for biosimilar/originator was assessed with four PCs' concentrations ranging from low to high.
Relative binding profiles (curves) were not compared, only the determined drug tolerance level for biosimilar and originator at each PC concentration level. The experiment was performed once. The determined drug concentrations for biosimilar/originator at all-tested PC concentration levels were within twofold of each other. Ideally, the drug tolerance (i.e., confirmed ADA to drug binding relative cut-point) should be similar (or within a close range) but due to variable nature of immunoassays, this is difficult to achieve even if the assay exhibits similar binding curves in previous experiments. The experiment demonstrated similar confirmed ADA (as significant response was cut-point dependent) binding as the drug tolerance profiles were comparable.

Relative binding curves with a high PC versus titrated spiked biosimilar/originator. Spiked responses were used for evaluation. Results were inconsistent (not dose linear) and a preparation error was suspected. The experiment was repeated once. Ocular assessment indicated curve similarity. This experiment was a repeat of development experiment (ii) but only performed at high PC level, as it provided the best dynamic range to evaluate any binding differences. Lessons learned and discussed; the development and validation testing provided a very comprehensive dataset, demonstrating the suitability of the assay to comparably detect antibodies against biosimilar and originator. However, the data were spread across a development report, and a validation report without an easy overview or clear conclusion for the reviewer. This could potentially be viewed as a regulatory risk in getting follow-up questions from regulators upon submission. Such a risk can be mitigated when preparing the integrated summary of immunogenicity by consolidating these data and this way improving overview and helping to follow through the set of experiments. For our subsequent programs, we implemented an improved strategy for documentation and topic transparency with clear conclusions and accessible data. Also, as biosimilar programs usually are extremely time sensitive and cost aware, the number of experiments performed, mainly in development, was re-evaluated. Some experiments were of critical value but quite a few turned out to provide limited extra value. In retrospect, a more lean approach could have been performed which would have yielded the same level of confidence in the oneassay approach. As a consequence, a more streamlined bioanalytical strategy (across programs), for demonstration of the one-assay approach, was also designed; see 'Case study 2: pegylated protein' for an example, and section 'Recommended approach for ADA binding comparability testing to justify the one-assay approach' for details. 


\section{Case study 2: pegylated protein}

The pharmaceutical drug type is a pegylated protein, consisting of an endogenous protein fused with a polyethylene glycol component. The indication is oncology. The bioanalytical strategy consisted of developing a tiered one-assay approach (labeled biosimilar was used for capture and detection) for ADA testing. Due to the frequency of dosing, drug tolerance was a concern; hence, the assay utilized the acid dissociation approach, where the sample was preincubated with the biosimilar. The platform was based on electrochemiluminescence. The PC used was generated by hyperimmunization of rabbits with the biosimilar. Routinely, each assay included three negative control samples and two low-concentration, two mid-concentration and two high-concentration PCs. All controls and samples were analyzed in duplicate. All comparisons were performed using biosimilar and originator US lot.

The below experiments (i) and (ii) were performed once during the late stages of development and once during validation, both times at the assay minimum required dilution. Ocular curve assessments were performed for both experiments. For experiment (ii), also a paired t-test was performed assessing mean difference.

Relative binding curves with low $/ \mathrm{mid} / \mathrm{high}$ PCs versus titrated spiked biosimilar/originator; see Figure 3.

Relative binding curves with titrated PC versus spiked confirmatory concentration of biosimilar/ originator were compared; see Figure 4.

This case study is a summary of the applied lesson learned from case study 1 and is representative of our recommended approach that is outlined and discussed in the following sections.

\section{Recommended approach for ADA binding comparability testing to justify the one- assay approach}

Designing a suitable bioanalytical strategy on how best to demonstrate the suitability of the one-assay approach is challenging. A careful balance is sought between required and unnecessary experiments. This is driven by time and cost constraints which are critical in biosimilar programs and, on the other side, submission- or regulatory hold-up risk. Figure 5 outlines an approach that we believe is balanced based on our experiences in the biosimilar industry.

The starting points of the outlined approach are the required reagents: biosimilar and originator drugs, labeled biosimilar as the core reagent and antibiosimilar antibody as PC. Once they are available, the assay development can start. Additionally, we recommend to have knowledge of the actual orig-

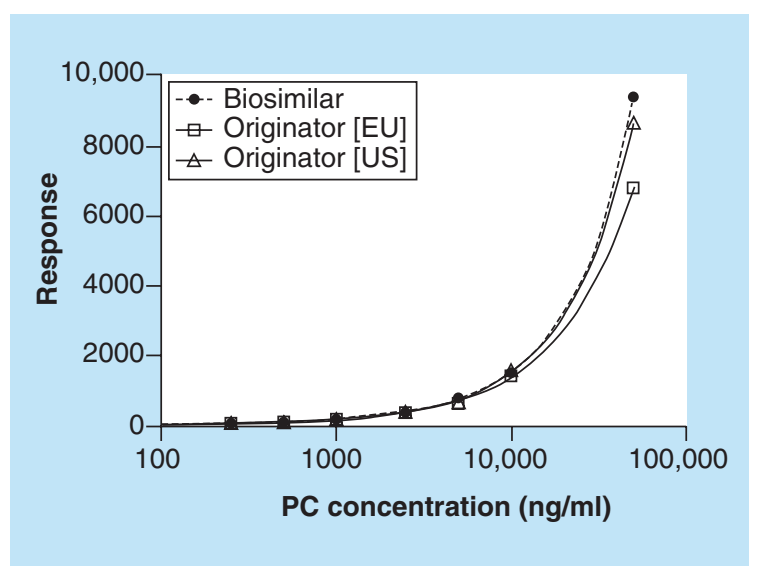

Figure 1. Relative binding curves with titrated positive control $(0-50,000 \mathrm{ng} / \mathrm{ml})$ versus spiked confirmatory concentration of biosimilar/originator (EU and US lots) $(100 \mu \mathrm{g} / \mathrm{ml})$ prepared in serum.

inator drug concentration, in other words, not just the label concentration. The actual drug concentration can either be found on the certificate of analysis if available or the originator should be analyzed using the same method that is being used for the biosimilar. During assay development, a basic one-assay feasibility testing should be performed. We propose, a minimum of two separate experiments, ideally performed once good estimates of PC concentration levels, minimum required dilution and confirmatory concentration are available.

The first experiment, 'titration comparability', is low PC and high PC signal inhibition curves obtained by titrating increasing concentrations of biosimilar and originator drugs. A suggested plate layout can be found in Figure 6. To evaluate the outcome of this experiment, the obtained curves should be compared using visual and/or statistical assessments. The aim is to demonstrate that the binding profiles of the PC toward the biosimilar and the originator are similar. It is noteworthy that the generated data can also be used for an early determination of the assay drug tolerance.

The second experiment, 'inhibitory comparability', consists of a titration of the PC at estimated confirmatory assay quenching concentrations of the biosimilar and the originator. If not yet determined, the quenching concentration should be as close as possible to the one that will be used during the future confirmatory assay. A suggested plate layout can be found in Figure 7. The aim of this experiment is to demonstrate that the assay is able to detect ADAs across a given antibody concentration range in a study representative confirmatory assay setup. The development feasibility testing should generate confidence in the one-assay approach. If data are borderline or not 
consistent, more experiments may be warranted. During validation, the same two experiments should be repeated in order to confirm the approach in a more controlled setting. For a robust assessment it is recommended to evaluate multiple data presentations of the comparability data, for example, response values and percentage of inhibition graphs. The totality of the bioanalytical data from development and validation should verify the consistency of the ADA binding comparability data. The two experiments offer two different perspectives of antibody-drug binding events and the combination of the data-sets should offer sufficient information to postulate a claim on the ADA binding comparability of the assay (i.e., oneassay justification).

The pros and cons of the recommended approach, when to perform the experiments and where to document the outcome will be discussed in the following sections. Note that the suggested plate layouts reflect a scenario where the biosimilar is tested with originator reference material from, for example, EU and USA. This is a common situation in biosimilar study design and assay comparability should be established with both originator reference lots.

\section{Documentation}

How is biosimilar assay suitability and ADA binding comparability testing best documented and where? Companies approach this differently; some include the experimental ADA binding comparability testing in validation while others have activities both in development and validation. Both are viable options,

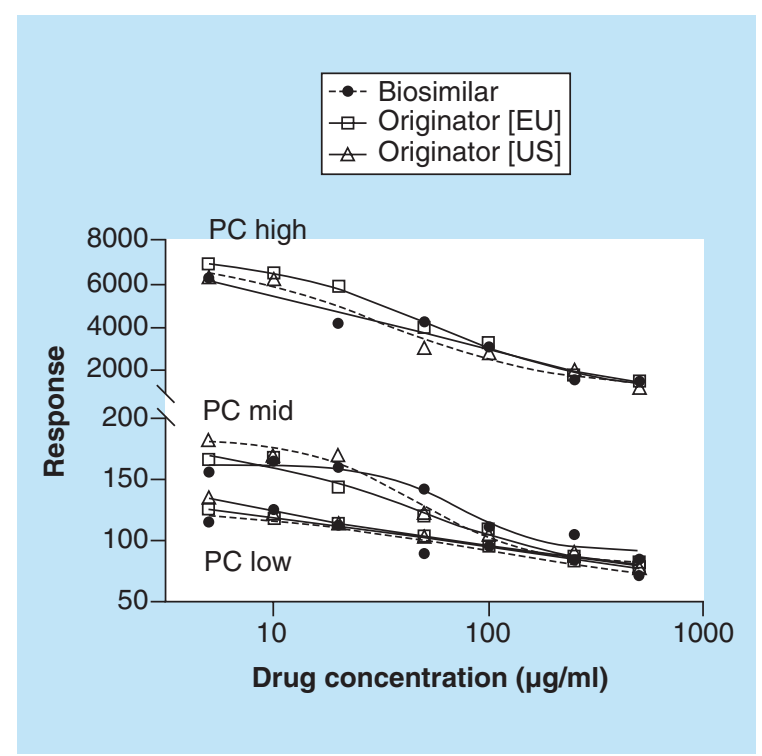

Figure 2. Relative binding curves with low/mid/high positive controls versus titrated spiked biosimilar/ originator (EU and US lots; $5-500 \mu \mathrm{g} / \mathrm{ml}$ ) prepared in serum. yet we advise to have a full understanding of the oneassay feasibility before going into validation. This does automatically constitute a situation where thorough documentation has to happen during development as well. In many situations, a formal assay development report may be excessive, but for biosimilars it does add value as a data repository supporting the one-assay suitability demonstration together with the data from validation. We recommend that the development phase includes experiments sensitive to operator errors, such as assessment of relative ADA binding profiles (using biosimilar and originator) as this will increase understanding of the method and increase confidence to move into validation phase.

Our recommendations for one-assay justification documentation are as follows:

- Add a dedicated section in the development report addressing 'assessment of one-assay suitability' (or similar). Compile or link relevant results there with a clear assessment and conclusion. Critical experiments should be documented in laboratory notebooks or equivalent during the development phase. Upon clarification, requests from regulators, data and notebooks need to be retrievable.

- Results supporting the suitability of the one-assay approach from validation should be summarized in the validation report under its own heading for transparency reasons and reviewer convenience. References to supportive data in the development report can be included. In this case, the development report also needs to be part of the submission dossier. If the comparability data should leave room for interpretation, a discussion section is needed to highlight relevance and potential impact of any observed differences.

- If an integrated summary of immunogenicity is prepared for the submission - which we strongly recommend - it should include a separate header for this topic where the suitability of the one-assay approach is summarized.

Due to the time-sensitive nature of a biosimilar's entry to market it is critical to design the bioanalytical submission package to be as complete as possible in order to meet the said critical timelines and also preemptive any questions regulators may have in order to not cause any delays for approval.

\section{Discussion}

Numerous factors, both reagent and parameter factors, have impact on the design of the bioanalytical 
biosimilar assay strategy. Here we discuss a few of these factors in conjunction with our recommended one-assay justification approach. High molecular analytical comparability is a prerequisite for its implementation.

The use of one assay or two assays for immunogenicity assessments has been widely debated in the bioanalytical community the past few years $[15,19,21]$. The pros and cons are briefly discussed in the above literature analysis. A notable published case example to support the use of the one-assay approach is Remsima ${ }^{\circledR}$ where both the one- and two-assay approaches were applied without any difference in outcome [22]. Based on our experiences in various biosimilar programs, we view using the biosimilar as labeling reagent and also as confirmatory quenching reagent in a one-assay approach as a conservative strategy suitable for a similarity immunogenicity exercise. The major criticism against this would be the risk of false negatives but only in a relative comparison setting. Using exclusively the biosimilar as areagent could be viewed as acceptable as it is the originator incidence which could then theoretically be underestimated which would magnify any differences between the molecules. It is also unclear if differences caused by epitopes unique for the originator and biosimilar would be evident in a two-assay setup. This approach suffers from numerous comparability constraints having potentially two sensitivities, two cut-points etc. It should be noted that biosimilar immunogenicity similarity assessments are usually not powered which could results in challenges when comparing, for example, the incidence between treatment arms. Immunogenicity 'equivalence' investigations benefit from clearly interpretable relative comparison datasets, and for this, the benefits of the one-assay approach far outweigh the two-assay approach.

Some papers have discussed the conservative approach of generating PC versus the biosimilar and originator separately in order to allow for a valid comparison of immunogenicity between the compounds [15,19]. Due to the surrogate nature of the polyclonal PCs and the high variability from immunization of animals, regardless of using same species or same immunization schedule, this approach may add significant imbalance to any comparability testing due to likely differences in affinity. The overall use of multiple PCs for any assay may result in potentially complex implications of having, for example, two sensitivities and/or two sets of acceptance criteria. As no reference sera (or a reference standard) exist for most biologics, a truly relevant comparison/ bridging reagent is impossible to obtain. No PC can

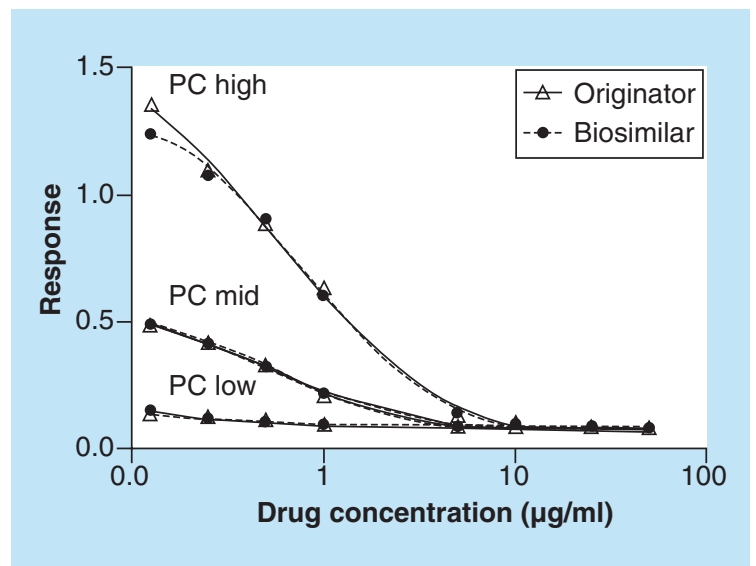

Figure 3. Relative binding curves with low/mid/high positive controls versus titrated spiked biosimilar/ originator $(0-60 \mu \mathrm{g} / \mathrm{ml})$ prepared in serum.

be expected to represent the spectrum of immune response observed in individuals [8]. For these reasons, we question the need to strive for full comparability between the PC immune spectrum and the population immune spectrum, as these can never be equal. Instead, the PC could be more viewed as a system suitability control and be treated as such. It is not uncommon to recommended that the PC be characterized for, for example, binding epitopes to confirm that the target binding patterns are reflective of normal immune response [14-15,19,23]. Due to the surrogate nature of PCs, our approach is to only perform baseline characterization, where the purity and specificity to the antigen are confirmed. No extra target epitope characterization is normally performed. Any activities and results related to immunization, purification and specificity to antigen are documented in a reagent characterization report or

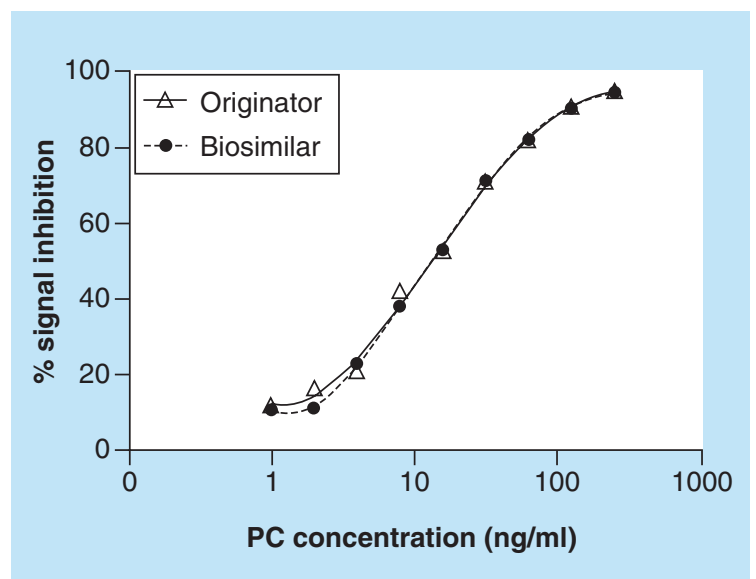

Figure 4. Relative binding curves with titrated positive control $(0-250 \mathrm{ng} / \mathrm{ml})$ versus spiked confirmatory concentration of biosimilar/originator $(50 \mu \mathrm{g} / \mathrm{ml})$ prepared in serum.

PC: Positive control. 
a certificate of analysis, depending on the extent of activities.

Overall, the options for the PC strategy can be described as:

- An antibiosimilar PC;
- Antibiosimilar and antioriginator PCs, where both have formal acceptance criteria;

- Antibiosimilar and antioriginator PCs, where only antibiosimilar PCs have formal acceptance crite-

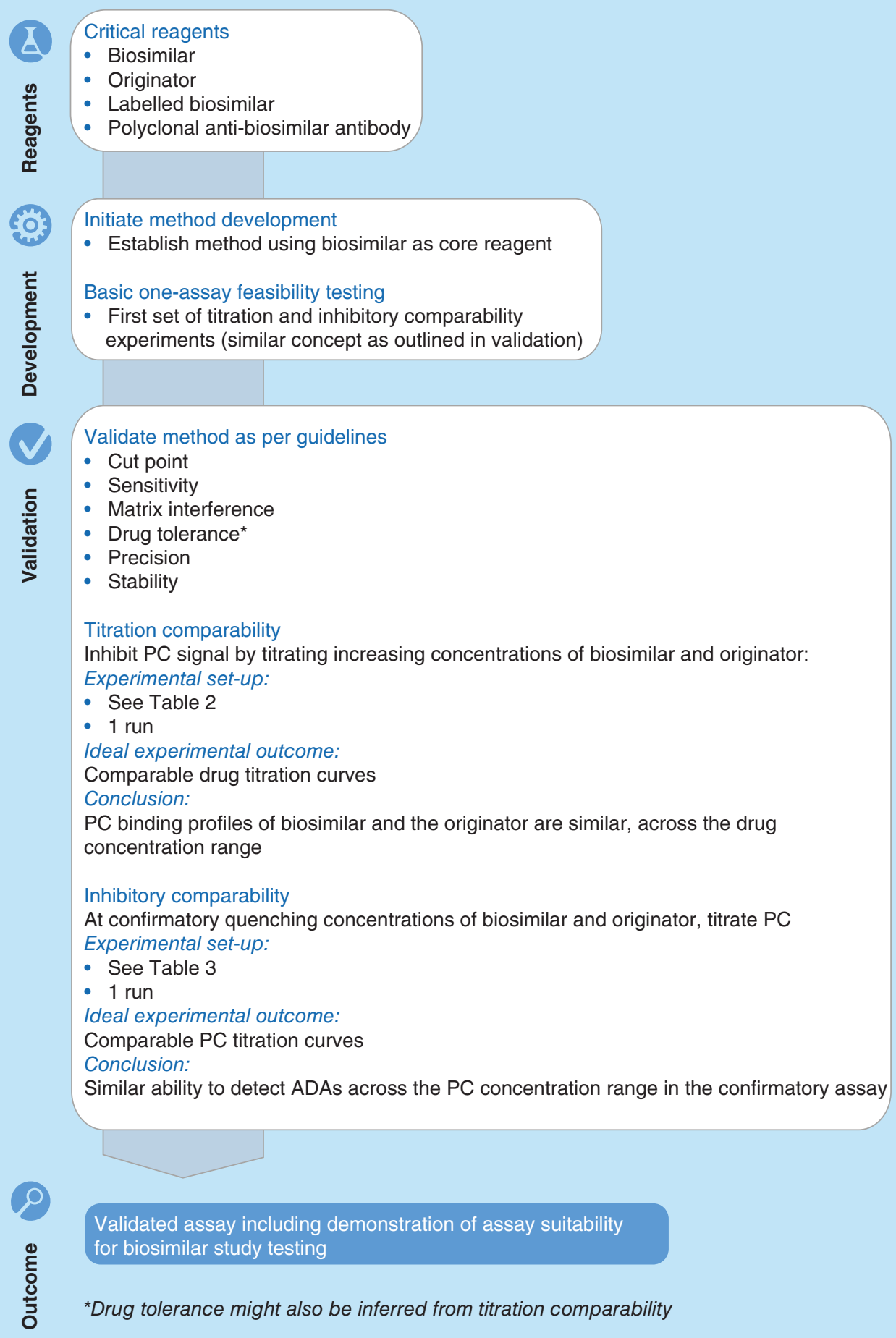

Figure 5. Recommended approach to one-assay suitability testing.

ADA: Antidrug antibody; PC: Positive control. 


\begin{tabular}{|c|c|c|c|c|c|c|c|c|c|c|c|c|c|}
\hline & & \multicolumn{2}{|c|}{ Biosimilar } & \multicolumn{2}{|c|}{ Originator (US) } & \multicolumn{2}{|c|}{ Originator (EU) } & \multicolumn{2}{|c|}{ Biosimilar } & \multicolumn{2}{|c|}{ Originator (US) } & \multicolumn{2}{|c|}{ Originator (EU) } \\
\hline & & 1 & 2 & 3 & 4 & 5 & 6 & 7 & 8 & 9 & 10 & 11 & 12 \\
\hline \multirow{8}{*}{ 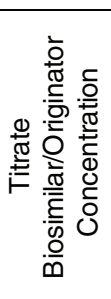 } & A & LPC & LPC & LPC & LPC & LPC & LPC & HPC & HPC & $\mathrm{HPC}$ & HPC & HPC & $\mathrm{HPC}$ \\
\hline & $\mathrm{B}$ & LPC & LPC & LPC & LPC & LPC & LPC & HPC & HPC & HPC & HPC & HPC & HPC \\
\hline & $C$ & LPC & LPC & LPC & LPC & LPC & LPC & HPC & HPC & HPC & HPC & HPC & HPC \\
\hline & $\mathrm{D}$ & LPC & LPC & LPC & LPC & LPC & LPC & HPC & HPC & HPC & HPC & HPC & $\mathrm{HPC}$ \\
\hline & $E$ & LPC & LPC & LPC & LPC & LPC & LPC & HPC & $\mathrm{HPC}$ & $\mathrm{HPC}$ & $\mathrm{HPC}$ & $\mathrm{HPC}$ & HPC \\
\hline & $\mathrm{F}$ & LPC & LPC & LPC & LPC & LPC & LPC & HPC & $\mathrm{HPC}$ & HPC & HPC & HPC & HPC \\
\hline & $\mathrm{G}$ & LPC & LPC & LPC & LPC & LPC & LPC & $\mathrm{HPC}$ & HPC & HPC & HPC & $\mathrm{HPC}$ & HPC \\
\hline & $\mathrm{H}$ & LPC & LPC & LPC & LPC & LPC & LPC & HPC & HPC & HPC & $\mathrm{HPC}$ & HPC & HPC \\
\hline
\end{tabular}

Figure 6. Example of plate layout for titration comparability experiment. If only EU or US originator is assessed, controls should be included. Alternatively one row could be substituted with controls.

HPC: High-concentration positive control; LPC: Low-concentration positive control.

ria and the antioriginator PCs are for monitoring purposes and cannot fail the assay;

- A commercial antibody binding the drug molecule (there may be reasons for not being able perform immunizations).

Due to various limitations of PC representativeness, and based on successful analytical comparability of biosimilar and originator, we apply a pragmatic approach and use a PC generated against biosimilar only. This option could be viewed as a regulatory conservative approach. We have to date not used commercial antibodies for the biosimilar programs, but would consider it if there was a time constraint. We are aware of cases [Pers. Сомm.] where commercial antibodies have been used successfully as PCs. The importance is to secure proper documentation and also the ensure supply of ideally the same batch throughout the clinical program.
As a future perspective to have better control of our system suitability controls, we are considering validating assays using both a polyclonal- and a monoclonal PC, both raised against the biosimilar. Validating with the polyclonal would ensure demonstration that a surrogate polyclonal immune response can be adequately detected whereas the monoclonal response would be used to argue that a monoclonal antibody can adequately works as a system suitability control. The main advantage of this approach is that the critical reagent life-cycle of the PC is more easily controlled using a monoclonal antibody, for example, easier to re-generate and ensure same reactivity/affinity than a polyclonal antibody.

What is critical to demonstrate in order to have full confidence in applying a one-assay strategy using the biosimilar? At minimum, it should be demonstrated that the assay can in a similar manner detect antibodies

\begin{tabular}{|c|c|c|c|c|c|c|c|c|c|c|c|c|c|}
\hline & \multirow[b]{2}{*}{1} & \multirow[b]{2}{*}{2} & \multicolumn{2}{|c|}{ Biosimilar confirm conc. } & \multicolumn{2}{|c|}{ Originator (US) confirm conc. } & \multicolumn{2}{|c|}{ Originator (EU) confirm conc. } & \multicolumn{2}{|c|}{ No drug } & \multirow[b]{2}{*}{11} & \multirow[b]{2}{*}{12} \\
\hline & & & & 3 & 4 & 5 & 6 & 7 & 8 & 9 & 10 & & \\
\hline \multirow{8}{*}{ 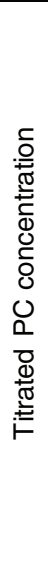 } & A & $\mathrm{NC}$ & NC & PC Conc 1 & PC Conc 1 & PC Conc 1 & PC Conc 1 & PC Conc1 & PC Conc 1 & $\begin{array}{c}\text { No } \\
\text { drug }\end{array}$ & $\begin{array}{c}\text { No } \\
\text { drug }\end{array}$ & & \\
\hline & B & NC & NC & PC Conc 2 & PC Conc 2 & PC Conc 2 & PC Conc 2 & PC Conc 2 & PC Conc 2 & $\begin{array}{c}\text { No } \\
\text { drug }\end{array}$ & $\begin{array}{c}\text { No } \\
\text { drug }\end{array}$ & & \\
\hline & C & LPC & LPC & PC Conc 3 & PC Conc 3 & PC Conc 3 & PC Conc 3 & PC Conc 3 & PC Conc 3 & $\begin{array}{c}\text { No } \\
\text { drug }\end{array}$ & $\begin{array}{c}\text { No } \\
\text { drug }\end{array}$ & HPC & $\mathrm{HPC}$ \\
\hline & $\mathrm{D}$ & LPC & LPC & PC Conc 4 & PC Conc 4 & PC Conc 4 & PC Conc 4 & PC Conc 4 & PC Conc 4 & $\begin{array}{c}\text { No } \\
\text { drug }\end{array}$ & $\begin{array}{c}\text { No } \\
\text { drug }\end{array}$ & HPC & $\mathrm{HPC}$ \\
\hline & $E$ & $\mathrm{HPC}$ & $\mathrm{HPC}$ & PC Conc 5 & PC Conc 5 & PC Conc 5 & PC Conc 5 & PC Conc 5 & PC Conc 5 & $\begin{array}{c}\text { No } \\
\text { drug }\end{array}$ & $\begin{array}{c}\text { No } \\
\text { drug }\end{array}$ & LPC & LPC \\
\hline & $\mathrm{F}$ & $\mathrm{HPC}$ & $\mathrm{HPC}$ & PC Conc 6 & PC Conc 6 & PC Conc 6 & PC Conc 6 & PC Conc 6 & PC Conc 6 & $\begin{array}{c}\text { No } \\
\text { drug }\end{array}$ & $\begin{array}{c}\text { No } \\
\text { drug }\end{array}$ & LPC & LPC \\
\hline & $\mathrm{G}$ & & & PC Conc 7 & PC Conc 7 & PC Conc 7 & PC Conc 7 & PC Conc 7 & PC Conc 7 & $\begin{array}{c}\text { No } \\
\text { drug }\end{array}$ & $\begin{array}{c}\text { No } \\
\text { drug }\end{array}$ & $\mathrm{NC}$ & $\mathrm{NC}$ \\
\hline & $\mathrm{H}$ & & & PC Conc 8 & PC Conc 8 & PC Conc 8 & PC Conc 8 & PC Conc 8 & PC Conc 8 & $\begin{array}{c}\text { No } \\
\text { drug }\end{array}$ & $\begin{array}{c}\text { No } \\
\text { drug }\end{array}$ & $\mathrm{NC}$ & $\mathrm{NC}$ \\
\hline
\end{tabular}

Figure 7. Example of plate layout for inhibition comparability experiment.

HPC: High-concentration positive control; LPC: Low-concentration positive control; NC: Negative control; PC: Positive control. 
directed against the biosimilar and originator. Our recommended comparability approach (Figure 5) evaluates this from two perspectives where the overall aim is to confirm, that in the assay, titrated ADAs can similarly bind to biosimilar/originator across a drug concentration range and also that ADA across a concentration range can similarly bind to biosimilars/originator at quench confirmatory concentration. We acknowledge that using the confirmatory quench concentration may be less sensitive to observing binding differences but this experiment is representative of the study sample analysis scenario and complements the data. Cai et al. recommend to assess drug tolerance for both drugs and with the criterion of an acceptable twofold to threefold difference [20]. If not met, the recommendation is to develop two assays. It should be evaluated if separate assessments of drug tolerance for biosimilars/originator adds more confidence in addition to the above relative binding experiments as drug tolerance assessment can be very variable. The same thinking can be applied for separate sensitivity assessments. If the above relative binding assessments can be considered similar, the argument can be made that the drug tolerance and sensitivity for biosimilar/originator are similar as well. Therefore, we recommend to only assess drug tolerance and sensitivity with the biosimilar.

A key gap in our recommendation is how the curve comparability is evaluated. Currently ocular assessment is applied as the baseline, but ideally a suitable unbiased preferably statistical approach should be used; however, this may require a larger dataset. Situations could arise where the ocular inspection yields inconclusive results, in other words, certain parts of the curves deviate. If this happens, it is advisable not to immediately conclude a mismatch, instead, to carefully review the experimental execution, evaluate impact and consider possible repeats in order to confirm the curve disparity. As ADA assays are not really quantitative, does it matter if, for example, the upper asymptote of the PC binding curve deviates? One could argue that in screening/confirmatory, it does not matter due to the qualitative nature of those assays. In the titer assay, however, there may be a risk of bias as the titer assessments are of a semiquantitative nature. The risk to biased data and clinical risk would need to be evaluated. The question remains, however: 'if the example of upper asymptote deviation would warrant the two-assay approach, which has its own challenges with biased data comparison'.

\section{Conclusion}

Presenting a solid bioanalytical package is critical in biosimilar drug development, in order to support PK and safety/efficacy trials. Current guidance documents do not address all the special considerations with bioanalytical assays used for similarity testing. Additional guidance on practical approaches is desirable. Our intent in this article is to provide one perspective on

\section{Executive summary}

\section{Background \& scope}

- One bioanalytical challenge of biosimilar drug development is how to demonstrate that the antidrug antibody (ADA) assay can adequately detect antibodies against both biosimilar and originator in the one-assay approach.

- The regulatory guidance on the practical conduct of immunogenicity studies in the biosimilar context is very limited and, so far, there is no consensus in the bioanalytical industry.

- A practical bioanalytical comparability testing approach is outlined and discussed.

Recommended approach for ADA binding comparability testing to justify the one-assay approach

- Biosimilar case studies are presented to provide a background for a recommended ADA comparability approach.

- The recommended comparability testing approach consists of two types of experiments: titration comparability - to demonstrate that the binding profiles of the PC toward the biosimilar and the originator are similar; and inhibitory comparability - to demonstrate that the assay is able to detect ADAs across a given antibody concentration range in a study representative confirmatory assay setup.

- The testing approach advocates an initial development feasibility phase, which should generate confidence in the one-assay approach. During validation, the same experiments should be repeated in order to confirm the approach in a more controlled setting.

\section{Documentation}

- Experiments supporting the one-assay approach should be documented in a dedicated section in the development report and/or validation report.

Future perspective

- Challenges remain for the bioanalytical industry to streamline the best practices for biosimilar ADA binding comparability testing.

- Near term: bioanalytical industry practices are moving toward the one-assay approach.

- Long term: availability of consolidated positive control set/panel for testing and comparison of biosimilars. 
how to perform bioanalytical ADA binding comparability testing to justify the one-assay approach. The provided recommended approach, herein, is derived from our experience in the field of biosimilars, feedback from regulators and shared experiences from external collaborators.

\section{Future perspective}

Many challenges remain for the bioanalytical industry to develop and consolidate the best practices for biosimilar ADA comparability testing. Ideally, the best practice is developed in collaboration with regulatory authorities. As more and more biosimilars reach the market, the knowledge base will increase with regulators and the bioanalytical industry. A possible scenario in the near term could be standardization of the one-assay approach. Thinking further ahead, an ideal development would be the availability of consolidated PC set/panel for testing and comparison of biosimilars.

\section{References}

Papers of special note have been highlighted as: $\bullet$ of interest;

•• of considerable interest

1 Declerck P, Danesi R, Petersel D, Jacobs I. The language of biosimilars: clarification, definitions, and regulatory aspects. Drugs 77(6), 671-677 (2017).

2 WHO. Guidelines on Evaluation of Similar Biotherapeutic Products (SBPs) (2009).

www.who.int/biologicals/areas/biological

3 European Medicines Agency. Guideline on Similar Biological Medicinal Products (2014).

www.ema.europa.eu/docs/en_GB/document_library

4 US FDA. Clinical Pharmacology Data to Support a Demonstration of Biosimilarity to a Reference Product (2016).

www.fda.gov/downloads/drugs/guidancecompliance

5 US FDA. Scientific Considerations in Demonstrating Biosimilarity to a Reference Product (2015). www.fda.gov/ downloads/drugs/guidances/ucm291128.pdf

6 Bui LA, Hurst S, Finch GL et al. Key considerations in the preclinical development of biosimilars. Drug Discov. Today 20 (Suppl. 1), 3-15 (2015).

7 Marini JC, Anderson M, Cai XY et al. Systematic verification of bioanalytical similarity between a biosimilar and a reference biotherapeutic: committee recommendations for the development and validation of a single ligand-binding assay to support pharmacokinetic assessments. AAPS J. 16(6), 1149-1158 (2014)

8 Shankar G, Devanarayan V, Amaravadi L et al. Recommendations for the validation of immunoassays used for detection of host antibodies against biotechnology products. J. Pharm. Biomed. Anal. 48(5), 1267-1281 (2008).

\section{Disclaimer}

The views and conclusion presented in this paper are those of the authors and do not necessarily reflect the representative affiliation or company's position on the subject.

\section{Financial \& competing interests disclosure}

The authors have no relevant affiliations or financial involvement with any organization or entity with a financial interest in or financial conflict with the subject matter or materials discussed in the manuscript. This includes employment, consultancies, honoraria, stock ownership or options, expert testimony, grants or patents received or pending, or royalties.

No writing assistance was utilized in the production of this manuscript.

\section{Open access}

This work is licensed under the Attribution-NonCommercialNoDerivatives 4.0 Unported License. To view a copy of this license, visit http://creativecommons.org/licenses/by-nc-nd/4.0/

9 European Medicines Agency. Guideline on Bioanalytical Method Validation (2012).

www.ema.europa.eu/docs/en_GB/document_library/

10 European Medicines Agency. Guideline on Immunogenicity Assessment of Monoclonal Antibodies Intended for in vivo Clinical Use (2012).

www.ema.europa.eu/docs/en_GB/document_library/

11 European Medicines Agency. Guideline on Immunogenicity Assessment of Biotechnology-Derived Therapeutic Proteins (2008).

www.ema.europa.eu/docs/en_GB/document_library

12 Food and Drug Administration. Assay Development and Validation for Immunogenicity Testing of Therapeutic Protein Products (2016).

www.fda.gov/downloads/Drugs/

13 US FDA. Bioanalytical Method Validation (2001). www.fda.gov/downloads/Drugs/Guidance/ucm070107.pdf

14 Richards S, Amaravadi L, Pillutla R et al. 2016 White Paper on recent issues in bioanalysis: focus on biomarker assay validation (BAV): (Part 3 - LBA, biomarkers and immunogenicity). Bioanalysis 8(23), 2475-2496 (2016).

- Publication proposing the one-assay approach as a consensus to assess immunogenicity of biosimilars.

15 Liu PM, Zou L, Sadhu C, Shen WD, Nock S. Comparative immunogenicity assessment: a critical consideration for biosimilar development. Bioanalysis 7(3), 373-381 (2015).

- Publication providing a comprehensive view of the pros and cons of the one-assay versus the two-assay approach to assess immunogenicity in the biosimilar context.

16 Islam R. Bioanalytical challenges of biosimilars. Bioanalysis 6(3), 349-356 (2014). 
17 Chamberlain P. Multidisciplinary approach to evaluating immunogenicity of biosimilars: lessons learnt and open questions based on 10 years' experience of the European Union regulatory pathway. Biosimilars 4, 23 (2014).

18 Chamberlain P. Assessing immunogenicity of biosimilar therapeutic monoclonal antibodies: regulatory and bioanalytical considerations. Bioanalysis 5(5), 561-574 (2013).

19 Cai XY, Thomas J, Cullen C, Gouty D. Challenges of developing and validating immunogenicity assays to support comparability studies for biosimilar drug development. Bioanalysis 4(17), 2169-2177 (2012).

20 Cai XY, Gouty D, Baughman S, Ramakrishnan M, Cullen C. Recommendations and requirements for the design of bioanalytical testing used in comparability studies for biosimilar drug development. Bioanalysis 3(5), 535-540 (2011).
21 Amaravadi L, Song A, Myler H et al. 2015 White Paper on recent issues in bioanalysis: focus on new technologies and biomarkers (Part 3 - LBA, biomarkers and immunogenicity). Bioanalysis 7(24), 3107-3124 (2015).

22 Reinisch W, Jahnsen J, Schreiber S et al. Evaluation of the cross-reactivity of antidrug antibodies to CT-P13 and infliximab reference product (remicade): an analysis using immunoassays tagged with both agents. Biodrugs 31(3), 223-237 (2017).

-. A case example where both the one-assay and two-assay approaches were applied and compared.

23 King LE, Farley E, Imazato $M$ et al. Ligand binding assay critical reagents and their stability: recommendations and best practices from the Global Bioanalysis Consortium Harmonization Team. AAPS J. 16(3), 504-515 (2014). 